\title{
A New Formula for Hemoglobin Level Prediction in Non-enhanced Brain Computed Tomography Scan
}

\author{
Mohammad Reza Sasani ${ }^{1}$, Yaser Toloueitabar (iD) ${ }^{2}$, Nahid Rezaeian $(\mathbb{D})^{2}$, Leila Hosseini (iD $^{2}$, Fatemeh \\ Zadehbagheri (i) ${ }^{2}$, Marzieh Motevalli ${ }^{2}$, Mahdi Daliri ${ }^{2}$ and Sanaz Asadian (i) ${ }^{1,2, *}$ \\ ${ }^{1}$ Medical Imaging Research Center, Department of Radiology, Shiraz University of Medical Sciences, Shiraz, Iran \\ ${ }^{2}$ Rajaie Cardiovascular Medical and Research Center, Iran University of Medical Sciences, Tehran, Iran \\ "Corresponding author: Rajaie Cardiovascular Medical and Research Center, Iran University of Medical Sciences, Tehran, Iran. Email: asadian_s@yahoo.com
}

Received 2020 April 20; Revised 2021 May 08; Accepted 2021 May 15.

\begin{abstract}
Background: For many patients with neurological complaints, a non-enhanced brain computed tomography (CT) scan is the first workup. In some of these patients, there is no pathological finding. Anemia is a condition that can present with neurological symptoms without any imaging findings. The correlation of dural venous sinus density with hemoglobin (Hb) level has been shown in some recent studies.

Objectives: This study aimed to propose a strategy to predict the level of $\mathrm{Hb}$ and investigate the possibility of underlying anemia based on dural venous sinus density to facilitate treatment.

Patients and Methods: The CT scans of selected patients, who were referred to Faghihi Hospital in Shiraz, Iran, from October 2018 until February 2019, were reviewed in this study; the complete blood count (CBC) was measured for cases without any findings. The data of 78 patients, including CBC parameters and the mean Hounsfield unit (HU) in the superior sagittal sinus (SSS), torcula herophili (TH), and transverse sinuses (TS), were also analyzed.

Results: A relatively strong direct linear correlation was found between the Hb level and HU. The Hb level was calculated based on the following formula: $\mathrm{Hb}$ level $=0.2 \times$ SSS HU $+1.2 \times$ sex factor $-0.01 \times$ age (where sex factor is zero for females and one for males). Besides, measurement of the cutoff point for the mean HU of SSS, based on the ROC curve to predict anemia, showed that with SSS $\mathrm{HU} \leq 50$, anemia could be predicted with $84.62 \%$ sensitivity, $75.38 \%$ specificity, and $75.64 \%$ accuracy in the general population.

Conclusion: A significant positive correlation was found between the $\mathrm{Hb}$ level and the mean $\mathrm{HU}$ of dural venous sinuses. Therefore, the level of $\mathrm{Hb}$ is predictable based on $\mathrm{HU}$, and differential diagnoses are limited.
\end{abstract}

Keywords: Cranial Venous Sinus Density, Hemoglobin, Brain Computed Tomography

\section{Background}

Today, non-enhanced brain computed tomography (CT) scan is performed as the first imaging step for most patients with neurological and psychological symptoms. However, in some of these patients, there are no pathological imaging findings to explain their signs and symptoms (1-3). One of the conditions presenting with neurological symptoms, without significant imaging findings, is anemia. It is the most common hematologic disorder, affecting 30\% of the world's population (4). This condition has non-specific symptoms, such as fatigue, palpitations, and exertional dyspnea $(4,5)$. It also causes neurological and psychological complaints, such as headache, poor concentration, and depression; even in the elderly, it is associated with a greater risk of Alzheimer's disease (6-9).

Recently, some studies have investigated the relation- ship between non-enhanced CT findings of different body areas and laboratory indicators of anemia. Recent studies have demonstrated the association of the myocardium, interventricular septum, and aortic wall density in nonenhanced chest CT scan with anemia $(10,11)$. Other investigations have reported a correlation between dural venous sinus density and blood hemoglobin ( $\mathrm{Hb}$ ) levels (1-3). If the association between dural venous sinus density and anemia is confirmed, patients with neurological or psychological complaints, without significant findings on nonenhanced brain CT scan, can be referred for further examinations and therapy, with the possibility of anemia as the underlying cause of their complaints. Although previous studies have demonstrated a significant correlation between dural venous sinus density and $\mathrm{Hb}$ level (1-3), further investigations are needed to determine its reproducibility in different populations. 


\section{Objectives}

In the present study, we aimed to propose a strategy to predict the $\mathrm{Hb}$ level and evaluate the possibility of anemia as an underlying cause based on the dural venous sinus density. Our findings can help identify anemia, improve the follow-up of patients, and improve their lifestyle.

\section{Patients and Methods}

In this cross-sectional study, 78 patients, who were referred for non-enhanced brain $\mathrm{CT}$ imaging to the Radiology Department of Shahid Faghihi Hospital in Shiraz, Iran, from October 2018 until February 2019, were evaluated. This study was approved by the Ethics Committee of Shiraz University of Medical Sciences, Shiraz, Iran. The exclusion criteria were as follows: age $\leq 18$ years; history of head trauma, intracranial hemorrhage, brain tumor, or cranial surgery; coagulopathy disorders; and an old or new cerebrovascular accident.

CT images were acquired with a Siemens Somatom Spirit Dual Slice Scanner, which was routinely calibrated using a calibration phantom. A non-enhanced brain CT scan was performed for each patient. The image acquisition parameters were as follows for all patients: a tube voltage of $130 \mathrm{kVp}$, a tube current of $110 \mathrm{mAs}$, and a slice thickness of $2.5 \mathrm{~mm}$ in an $8-\mathrm{mm}$ slice interval. The mean volume CT dose index was $25.05 \mathrm{mGy}$ (range: $22-27.50 \mathrm{mGy}$ ). The images were reviewed immediately by an expert radiologist in a window level of 35 Hounsfield units (HU) and window width of $80 \mathrm{HU}$. Patients without pathologic imaging findings were asked to sign an informed consent form and were referred for blood sampling to determine the complete blood count (CBC). Checklists were used to register the clinical and laboratory parameters accurately.

The acquired images were transferred from INFINITT PACS to eFilm Workstation 2.1 Merge eMed to designate the HU. An expert radiologist, unaware of the laboratory parameters, interpreted the imaging studies to reduce observer bias. The region of interest (ROI) was selected in sites with minimum beam hardening artifact. For all examinations, the ROI area was $0.1 \mathrm{~cm} 2$. HU was measured at the level of torcular herophili $(\mathrm{TH})$ (one section above and one section below it). The mean $\pm S D$ of $\mathrm{HU}$ was recorded as mean \pm SD HU of TH for the mentioned three sites. Also, $\mathrm{HU}$ was measured in three consecutive sections in the posterior (more apparent) part of the superior sagittal sinus (SSS) (Figure 1 presents the measurement of $\mathrm{HU}$ at the level of SSS), as well as bilateral transverse sinus (TS) in the same segment. In case of unilateral hypoplasia of TS, HU was measured in normal sinuses in two consecutive sections.
The mean \pm SD HU was measured for three SSS measurements, and also, two or three TS HU values were calculated and recorded for each patient in the checklists.

\subsection{Statistical Analysis}

Data were entered in SPSS version 18 for data analysis. Categorical variables are presented as frequency and percentage, while continuous variables are reported as mean \pm SD. Kolmogorov-Smirnov test was used for assessing and confirming the normal distribution of variables, and Pearson's correlation test was applied for quantitative variables. Based on student's t-test, a significant correlation ( $\mathrm{P}$ $<0.01$ ) was found between sex and Hb level. Next, to determine independent factors influencing the $\mathrm{Hb}$ level, multiple linear regression analysis was carried out. Age, sex, mean $\mathrm{HU}, \mathrm{WBC}$, and platelet count were also investigated in this study. Moreover, the stepwise method was applied to develop a regression model; the characteristics of the model are presented in Table 1. Finally, a receiver operating characteristic (ROC) curve analysis was performed to determine the cutoff point and estimate sensitivity, specificity, and accuracy. P-values $<0.05$ were considered statistically significant.

\begin{tabular}{lccc}
\hline \multicolumn{4}{l}{ Table 1. Variables of the Regression Model and Their Coefficients ${ }^{\mathrm{a}, \mathrm{b}}$} \\
\hline Variables & B & SE & P-value \\
\hline Constant & 3.342 & 1.988 & 0.097 \\
SSS & 0.2 & 0.039 & 0.000 \\
Sex & 1.2 & 0.267 & 0.000 \\
Age & -0.01 & 0.006 & 0.036 \\
\hline
\end{tabular}

Abbreviations: SSS, superior sagittal sinus; SE, standard error.

${ }^{\text {a }}$ Model R2: 0.579 .

${ }^{\mathrm{b}}$ Dependent Variable: Hemoglobin.

\section{Results}

Seventy-eight patients, including 23 men (29.5\%) and 55 women $(70.5 \%)$ in the age range of $21-83$ years $($ mean $=$ $43.44, \mathrm{SD}=16.5$ ), entered this study. The mean \pm SD HU was measured as previously described for each dural sinus. It was found that SDs are constant with a narrow range of change (2.7-4) in all examined sites; therefore, they did not influence the results. Moreover, scatter plots were drawn to evaluate the correlation of $\mathrm{Hb}$ level with the mean $\mathrm{HU}$ in each mentioned site and also with age. Figure 2 indicates the scatter plot of the correlation of $\mathrm{Hb}$ level with the mean SSS HU. According to the scatter plots, there was a significant positive correlation between the level of $\mathrm{Hb}$ and the mean HU in each site. Table 2 presents the results of Pearson's correlation test. 

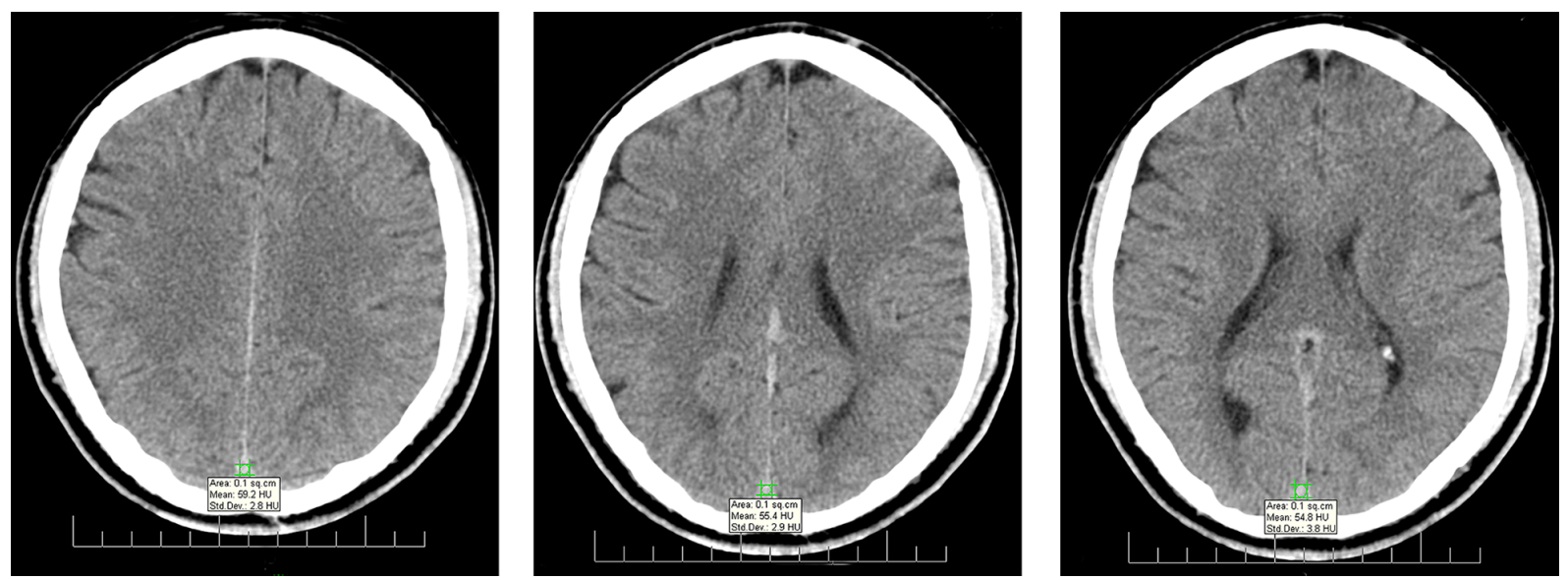

Figure 1. Measurement of Hounsfield unit (HU) at the level of superior sagittal sinus (SSS)

\begin{tabular}{lccc}
\hline \hline Table 2. The Results of Pearson's Correlation Test & & \\
\hline Hb & SSS HU & TH HU & TS HU \\
\hline Pearson's correlation coefficient & 0.665 & 0.591 & 0.535 \\
P-value & 0.000 & 0.000 & 0.000 \\
\hline
\end{tabular}

Abbreviations: SSS, superior sagittal sinus; HU, Hounsfield unit; TH, torcula herophili; TS, transverse sinuses; Hb, hemoglobin.

There was a significant direct relationship between the level of $\mathrm{Hb}$ and the mean $\mathrm{HU}$ in SSS, TH, and TS, with correlation coefficients of $0.66,0.59$, and 0.53 , respectively $(\mathrm{P}<$ 0.0001 for all). In our study, the most significant correlation was found between the level of $\mathrm{Hb}$ and the mean $\mathrm{HU}$ in SSS (correlation coefficient: 0.66). Also, the results of Pearson's correlation test revealed the colinearity of the mean HU in our three sites of measurement. Therefore, the SSS HU was used for further analyses. Also, a weak reverse linear correlation was found between the $\mathrm{Hb}$ level and age $(\mathrm{P}$ $=0.06, \mathrm{r}=-0.20)$. Besides, a significant correlation was observed between sex and Hb level $(\mathrm{P}<0.01)$. Next, a multiple linear regression analysis was performed. By studying age, sex, the mean HU, WBC, and platelet count based on a stepwise approach, a regression model was extracted. The $\mathrm{Hb}$ level was predicted based on the following formula:

$\mathrm{Hb}$ level $=0.2 \times$ SSS HU $+1.2 \times$ sex factor $-0.01 \times$ age

where sex factor is zero for females and one for males. With each unit increase in SSS HU, the Hb level increased by $0.2 \mathrm{~g} / \mathrm{dL}$ in each patient. According to the definition of anemia ( $\mathrm{Hb}<14 \mathrm{~g} / \mathrm{dL}$ in males and $\mathrm{Hb}<12 \mathrm{~g} / \mathrm{dL}$ in females $), 12$ $(15.4 \%)$ patients, including two men and ten women, were anemic. The mean, median, and frequency of SSS HU in anemic and non-anemic men and women are shown in box plots. Figure 3 presents a box plot of SSS HU among women in anemic and non-anemic groups.

A significant correlation was found between anemia and the mean $\mathrm{HU}$ in all three sites $(\mathrm{P}<0.05)$, and the mean HU was significantly higher in the non-anemic group. The ROC curve analysis revealed that at a cut-off point of SSS $\mathrm{HU} \leq 50$, anemia could be predicted in the general population with sensitivity of $84.62 \%$, specificity of $75.38 \%$, and accuracy of $75.64 \%(P=0.0001, A U C=0.8)$. In the subgroup ROC curve analyses for men and women, a significant, but weak result was obtained in the male group $(P=0.54$, AUC $=0.69$ ), while the result showed strong significance in the female group $(\mathrm{P}=0.0005$, AUC $=0.77)$. Therefore, by considering the SSS $\mathrm{HU} \leq 50$ as the cutoff point, anemia could be predicted in the female group with sensitivity of $90.91 \%$, specificity of $63.64 \%$, and accuracy of $67.27 \%$.

\section{Discussion}

Recently, several studies have been performed to assess non-enhanced CT images of different parts of the body and laboratory findings of anemia, especially the $\mathrm{Hb}$ level. Some studies confirmed the significant association of dural venous sinus density with the blood Hb level (13). If such a relationship is confirmed, patients with neurological symptoms without significant findings on nonenhanced brain CT imaging can be referred for further workup and therapy. In our study, a significant positive correlation was observed between the level of $\mathrm{Hb}$ and the mean HU in each of the three examined sites (SSS, TH, and TS). A weak negative correlation was found between the $\mathrm{Hb}$ level and age; also, there was a significant relationship between the level of $\mathrm{Hb}$ and sex. A formula was extracted based on the multiple regression analysis to predict the $\mathrm{Hb}$ level. 


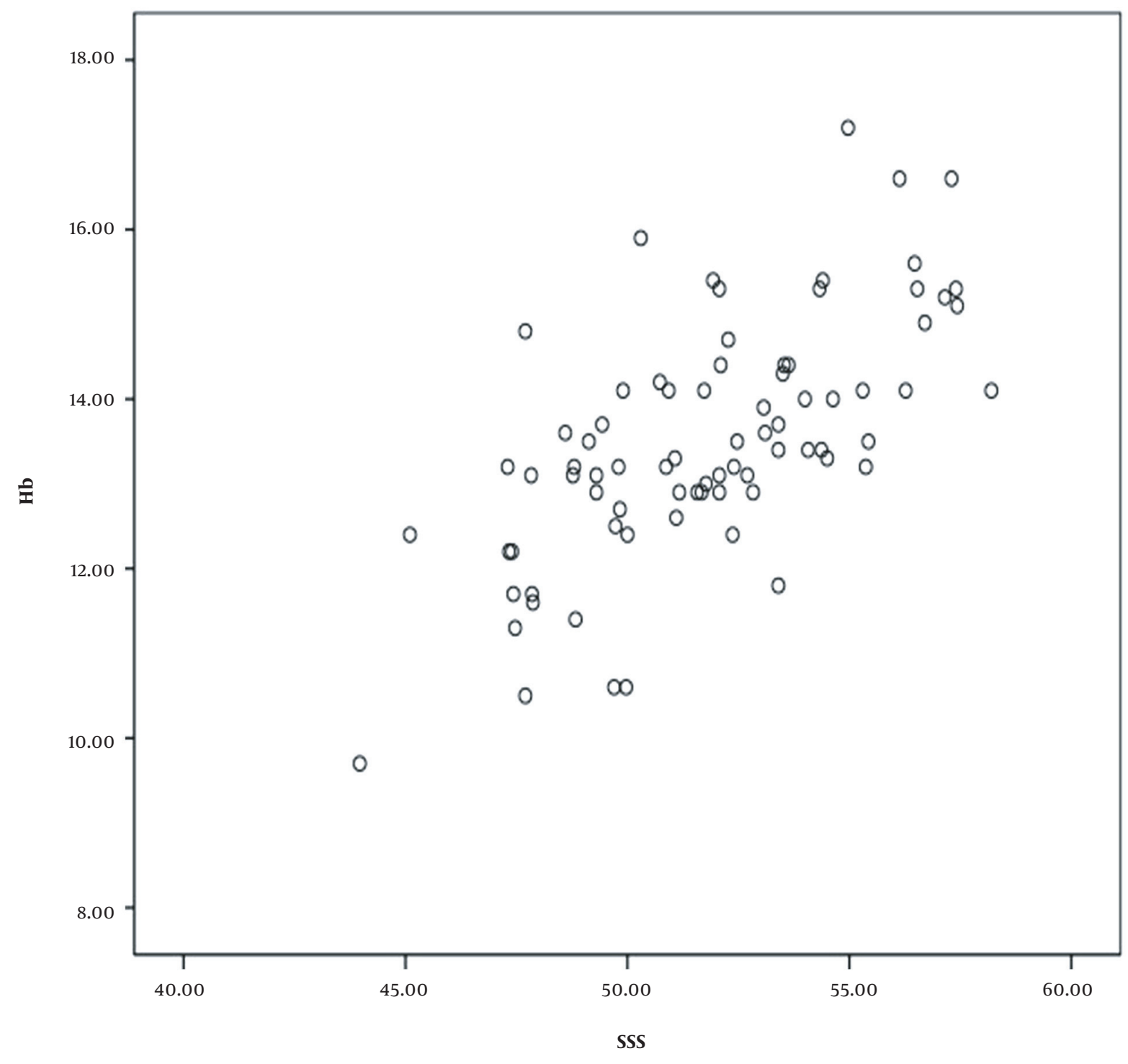

Figure 2. The scatter plot of the correlation of hemoglobin (Hb) level with the mean superior sagittal sinus (SSS) Hounsfield unit (HU)

According to our results, SSS (with the highest correlation coefficient) was the most appropriate site for $\mathrm{Hb}$ prediction among the three examined sites. Because of the colinearity of the mean HU in these three sites, only SSS HU was used for subsequent assessments. Three variables, including age, sex, and SSS HU, could predict the $\mathrm{Hb}$ level. Meanwhile, the role of SSS HU in prediction was more prominent, and the SSS cutoff point was measured to be $\leq 50 \mathrm{HU}$ in the general and female populations with acceptable sensitivity.

Our results are comparable to several previous studies. Nevertheless, it should be noted that we excluded patients younger than 18 years as a confounding variable due to dynamic brain changes in adolescents. The correlation coefficients measured in our study are more significant than those reported by Bruni et al., while they are lower than the results obtained by Seung Young Lee et al. (3); therefore, we can assume that our results have a remarkable influence. For accurate data registration, we used face-to-face communication with the patients and performed $\mathrm{CBC}$ within the shortest possible interval from the imaging study $(<1$ h).

In most previous studies, no comparison was made to find the best site of dural venous sinuses for measuring $\mathrm{HU}$ 


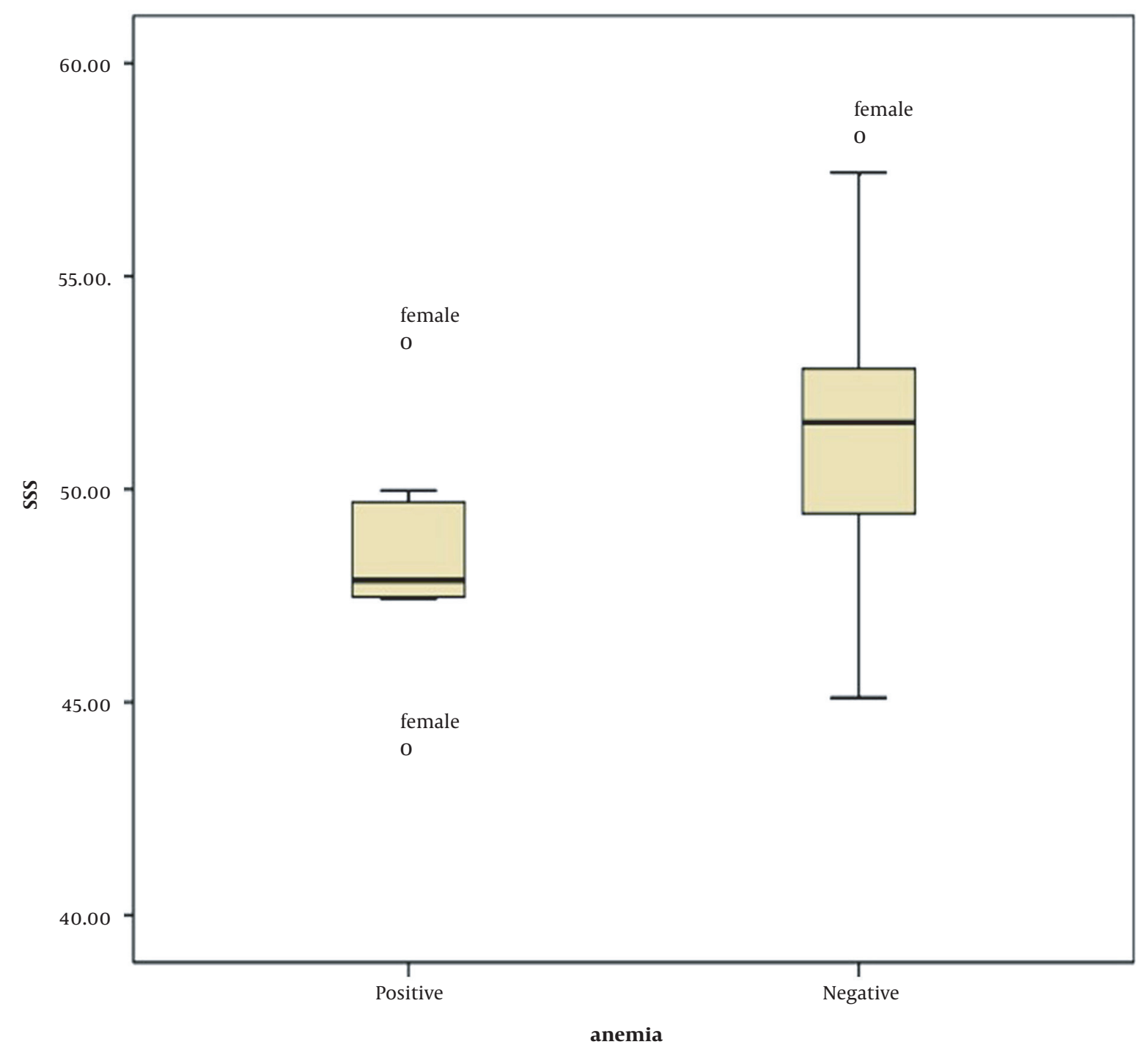

Figure 3. The box plot of superior sagittal sinus (SSS) Hounsfield unit (HU) among females in the anemic and non-anemic groups

to predict the $\mathrm{Hb}$ level. To the best of our knowledge, this study is one of the few investigations regarding the importance of dural venous sinus measurement sites. Similar to a study by Lee et al., we surveyed HU in several sections and used the mean values for analysis, which increased the accuracy of our measurements (2). Our study is possibly one of the few investigations that implemented a multiple regression analysis to extract a mathematical formula for predicting the $\mathrm{Hb}$ level, based on the SSS HU, sex, and age. Similar formulae for the female and male groups were obtained in a study by Bruni and colleagues (2). Nevertheless, we believe that our formula is more applicable in routine practice by radiologists.

In this regard, Chaudhry et al. examined 243 patients, undergoing non-enhanced brain CT imaging in a tertiary care center as part of a designated workup to find the neurological cause of their complaints. They classified the patients into four categories based on the Hb level. The mean $\mathrm{HU}$ values were recorded for dural venous sinuses. Contrary to our findings, the most significant positive correlation was found between the Hb level and the confluence of sinus density. A marked similarity between our findings and those reported by Chaudhry et al. is the correlation coefficient measurement. The Pearson's correlation coeffi- 
cient for the correlation of SSS HU with Hb level was 0.66 in our study. Similarly, Chaudhry et al. reported R2 of 0.63 for the correlation of $\mathrm{HB}$ with attenuation at the confluence of dural sinuses. By conducting further research, more reliable results can be obtained regarding the measurement of dural venous sinus density for the prediction of $\mathrm{Hb}$ level (12).

Moreover, another large-scale study $(n=640)$ indicated that among parameters influencing the dural venous sinus attenuation, $\mathrm{Hb}$ and hematocrit levels were the only factors with significant correlations with the average dural sinus attenuation (13). A similar investigation of 230 individuals ( 54 cases with cerebral intravascular clot and 176 cases without thrombus) revealed that hematocrit, red blood cell count, and $\mathrm{Hb}$ level were the main determinants of venous sinus density. They also emphasized that the differences between the right and left intracranial vessels were more reliable for identifying clots as compared to the HU value alone (14). These findings support our results and reinforce the suggestion of using dural venous sinus density to estimate the Hb level.

Among different body parts recently investigated for $\mathrm{Hb}$ level prediction, dural venous sinuses are the most frequently mentioned sites. The results of several studies evaluating other body parts are also consistent with the findings of cerebral venous sinuses. The study conducted by Foster et al. may be one of the oldest studies to estimate anemia based on non-enhanced CT characteristics qualitatively. They concluded that the visibility of the interventricular septum on a non-enhanced thoracic CT scan is suggestive of underlying anemia (10). Another example is a case-report by Marmol et al., using sites other than dural venous sinuses to determine anemia. They described a case of gastrointestinal bleeding with a concomitantly decreased density of dural venous sinuses, abdominal aorta, vena cava, and cardiac chambers (15).

In a prominent study on 317 patients with different severity classes of anemia (mild, moderate, and severe), the difference between the HU value of the interventricular septum and the left ventricle cavity (IVS-LV) was calculated, and a strong correlation with the Hb level was found. Similarly, we found a direct linear correlation between the mean HU value in all of the examined dural venous sinus sites and Hb level. They also measured the IVS-LV cutoff points in different severity subgroups for the diagnosis of anemia (16).

One of our study limitations was the small male sample size (23 men vs. 55 women). Therefore, we could not reliably determine the cutoff point in the male population, and the measured cutoff point for the female group was relatively close to the general population (50 HU). It appears that further research on a larger sample size and comparable male-to-female ratios is needed to determine more precise cutoff points for the general community and gender subgroups. Also, some previous studies have reported inter-scanner variations in HU measurements. In a study by Lamba et al., different CT measurements were reported for abdominal soft tissues, using two distinct common CT scanners (17). Likewise, Oh et al. found that the gray and white matter-calculated HUs, and consequently their ratios, were strikingly variable among different CT scan systems (18). Besides, in some studies, variations between the measured HUs at different energy levels of radiation have been reported (19). Therefore, our developed formula should be cautiously used in other CT machines unless it is modified in future studies.

In conclusion, a significant positive correlation was found between the Hb level and the mean HU of dural venous sinuses; therefore, the level of $\mathrm{Hb}$ is predictable based on HU, and differential diagnoses are limited. However, determining a cutoff point for $\mathrm{HU}$ to predict anemia requires further research.

\section{Footnotes}

Authors' Contribution: Study concept and design: M.S. and S.A.; Analysis and interpretation of data: Y.T., and N.R.; Drafting of the manuscript: L.H.; Critical revision of the manuscript for important intellectual content: F.Z. and M.M.; and Statistical analysis: M.D.

Conflict of Interests: The authors declare no conflict of interest.

Ethical Approval: Code: Ir.sums.rec.1393.5519. Date of ethical approval: 18.05.1393.

\section{Funding/Support: None.}

Informed Consent: The author (Sanaz Asadian as the corresponding author of "A new formula for hemoglobin level prediction in non-enhanced brain computed tomography scan") declares that informed consent was obtained from all patients included in this study.

\section{References}

1. Black DF, Rad AE, Gray LA, Campeau NG, Kallmes DF. Cerebral venous sinus density on noncontrast CT correlates with hematocrit. Am. J. Neuroradiol. 2011;32(7):1354-7. doi: 10.3174/ajnr.A2504.

2. Bruni SG, Patafio FM, Dufton JA, Nolan RL, Islam O. The assessment of anemia from attenuation values of cranial venous drainage on unenhanced computed tomography of the head. Can Assoc Radiol J. 2013;64(1):46-50. doi:10.1016/j.carj.2011.08.005.

3. Lee SY, Cha S, Lee S, Shin D. Evaluation of the effect of hemoglobin or hematocrit level on dural sinus density using unenhanced computed tomography. Yonsei Med.J. 2013;54(1). doi:10.3349/ymj.2013.54.1.28.

4. Parker-Williams EJ. Investigation and management of anaemia. FY. 2007;3(2):86-91. doi: 10.1016/j.mpfou.2007.01.009. 
5. Braunwald E, Fauci AS, Kasper DL, Hauser SL, Longo DL, Jameson JL. Harrison's principles of internal medicine. McGraw Hill; 2001.

6. Corwin EJ, Murray-Kolb LE, Beard JL. Low hemoglobin level is a risk factor for postpartum depression. J. Nutr. 2003;133(12):4139-42. doi: 10.1093/jn/133.12.4139.

7. GuralnikJM, Ershler WB, Schrier SL, Picozzi VJ. Anemia in the elderly: A public health crisis in hematology. Hematology. 2005;2005(1):528-32. doi: 10.1182/asheducation-2005.1.528.

8. Pandav RS, Chandra V, Dodge HH, DeKosky ST, Ganguli M Hemoglobin levels and alzheimer disease: An epidemiologic study in India. Am J Geriatr Psychiatry. 2004;12(5):523-6. doi:10.1097/00019442 200409000-00011.

9. Thein M, Ershler WB, Artz AS, Tecson J, Robinson BE, Rothstein G, et al. Diminished quality of life and physical function in communitydwelling elderly with anemia. Medicine. 2009;88(2):107-14. doi: 10.1097/MD.ob013e31819d89d5.

10. Foster M, Nolan RL, Lam M. Prediction of anemia on unenhanced computed tomography of the thorax. Can Assoc Radiol J. 2003;54(1):26-30. [PubMed: 12625080]

11. Jung C, Groth M, Bley TA, Henes FO, Treszl A, Adam G, et al. Assessment of anemia during CT pulmonary angiography. Eur. J. Radiol. 2012;81(12):4196-202. doi: 10.1016/j.ejrad.2012.07.008.

12. Chaudhry AA, Gul M, Chaudhry A, Sheikh M, Dunkin J. Quantitative evaluation of noncontrast computed tomography of the head for assessment of anemia. J. Comput. Assist. Tomogr. 2015;39(6):842-8. doi: 10.1097/rct.0000000000000306.
13. Akhavan R, Abbasi B, Kheirollahi M, Ghamari Khameneh A, Hashemi $\mathrm{J}$, Khoei $\mathrm{S}$, et al. Factors affecting dural sinus density in noncontrast computed tomography of brain. Sci. Rep. 2019;9(1). doi: 10.1038/s41598-019-48545-y.

14. Grams AE, Rehwald R, Schmittnägel C, Schmidt T, Tanislav C, Berghoff $M$, et al. Factors influencing intracranial vessel densities on unenhanced computed tomography: differences between hemispheres. Clin. Imaging. 2016;40(6):1081-5. doi:10.1016/j.clinimag.2016.06.012.

15. del Marmol O, Coulier B. "Black" and "white" blood on unenhanced CT. J. Belgian Soc. Radiol. 2019;103(1). doi:10.5334/jbsr.1733.

16. Zhou Q, Yu Y, Chen Y, Ding B, Fang S, Yang X, et al. Optimal threshold for the diagnosis of anemia severity on unenhanced thoracic CT: A preliminary study. Eur. J. Radiol. 2018;108:236-41. doi: 10.1016/j.ejrad.2018.10.007.

17. Lamba R, McGahan JP, Corwin MT, Li C, Tran T, Seibert J, et al. CT hounsfield numbers of soft tissues on unenhanced abdominal CT scans: Variability between two different manufacturers' MDCT scanners. Am. J. Roentgenol. 2014;203(5):1013-20. doi: 10.2214/ajr.12.10037.

18. Oh $\mathrm{JH}$, Choi SP, Wee $\mathrm{JH}$, Park JH. Inter-scanner variability in Hounsfield unit measured by CT of the brain and effect on grayto-white matter ratio. Am. J. Emerg. Med. 2019;37(4):680-4. doi: 10.1016/j.ajem.2018.07.016.

19. Bolus D, Morgan D, Berland L. Effective use of the Hounsfield unit in the age of variable energy CT. Abdom. 2017;42(3):766-71. doi: 10.1007/s00261-017-1052-4. 